\title{
The threshold elicited motor response via an in situ femoral nerve catheter predicts analgesia following total knee replacement
}

\author{
Antoun Nader \\ Mark C Kendall \\ Brian Chung \\ Kiran Chekka \\ Khalid Malik \\ Robert J McCarthy \\ Department of Anesthesiology, \\ Northwestern University Feinberg \\ School of Medicine, Chicago, IL, USA
}

Correspondence: Mark C Kendall Department of Anesthesiology, 25 I E. Huron, F5-704, Chicago, IL 606 I I, USA Tel + I 3/29265278

Fax + I 312926834 I

Email m-kendall@northwestern.edu
This article was published in the following Dove Press journal:

Local and Regional Anesthesia

14 September 2010

Number of times this article has been viewed

Background: Continuous femoral nerve blocks are a part of the multimodal perioperative anesthetic regimen following total knee replacement. Elicitation of a quadriceps muscle contraction (QC) at placement is desirable. We prospectively evaluated the relationship between elicited motor response and threshold current with block success in situ femoral nerve catheters after total knee replacement.

Methods: After Institutional Review Board approval and written informed consent, 100 adults aged $\geq 18$ years, undergoing total knee replacement were studied. The threshold current for an elicited motor response (QC or sartorius muscle contraction [SC]) was recorded during needle insertion, after femoral nerve catheter advancement, and prior to local anesthetic administration on the first postoperative day. Patients were assessed for pinprick sensory anesthesia of the femoral nerve distribution by an observer unaware of the current threshold or evoked motor response at 5-minute intervals for 30 minutes. A successful block was considered a lack of pinprick sensation within 30 minutes.

Results: Ninety patients completed the study. A QC was obtained at needle insertion in 89 and maintained following catheter advancement in 77 subjects. Prior to drug administration a QC was obtained in 66 patients, 13 demonstrated an SC, and 11 had no motor response. QC prior to drug administration had a sensitivity of 0.85 (95\% confidence intervals [CI]: 0.75 to 0.92$)$ and a specificity of 0.55 ( $95 \%$ CI: 0.37 to 0.72 ) for complete femoral nerve block. The specificity of a QC prior to drug administration was greater than at catheter placement $(P=0.006)$. Compared to the threshold current at catheter placement, 27 patients had a decrease, 60 had an increase, and 3 exhibited no change in the threshold current prior to drug administration $(P<0.01)$. The median interquartile range (IQR) threshold current in patients that achieved a complete block was $0.56(0.29$ to 0.80$) \mathrm{mA}$ compared with $1.1 \mathrm{~mA}(0.41$ to 2.75$) \mathrm{mA}$ for incomplete blocks $(P<0.01)$. The area under the receiver operator characteristics curve for current threshold prior to local anesthetic administration $(0.74)$ was greater than at catheter placement $(0.45)$ $(P<0.001)$. The intersection of sensitivity and specificity for the minimal threshold current prior to local anesthetic injection was $0.84 \mathrm{~mA}$.

Conclusion: The elicited motor response and current threshold from a stimulating femoral catheter measured prior to local anesthetic injection is an important determinant of the success of femoral nerve block following bolus administration.

Keywords: stimulating femoral nerve catheters, total knee replacement, postoperative analgesia

\section{Introduction}

Continuous femoral nerve blockade via an indwelling catheter has been used as part of a multimodal analgesic management protocol for patients undergoing knee surgery. ${ }^{1}$ 
These catheters are placed perioperatively and are frequently maintained in place unused for many hours without subsequent reconfirmation of the catheter tip location. When using nerve stimulator assistance to place these catheters, an elicited motor response (EMR) of a quadriceps contraction (QC) at a defined current threshold is frequently sought after. $^{2}$ Although the initial current threshold and evoked motor response are determinates of the block success when local anesthetic is administered immediately, migration of catheters and edema in surrounding tissues can result in a different EMR or threshold current at subsequent times. We prospectively evaluated the influence of the initial current threshold and EMR obtained during placement of stimulating femoral catheters on subsequent EMRs, current thresholds and success rates on postoperative day one (POD 1) in 100 patients undergoing total knee replacement.

\section{Methods}

After Institutional Review Board approval and written informed consent, adults aged $\geq 18$ years, undergoing total knee replacement, American Society of Anesthesiologists physical status I-III, were eligible for participation in this study. Exclusion criteria included: contraindication to epidural or spinal anesthesia, coagulopathy, infection or previous surgery at the site of epidural or femoral catheter placement, or preexisting neurologic dysfunction. All patients received neuraxial anesthesia with intraoperative sedation. In the post anesthesia recovery area, patient controlled epidural analgesia with an infusion containing bupivacaine $1 \mathrm{mg} / \mathrm{mL}$ and hydromorphone $10 \mu \mathrm{g} / \mathrm{mL}$ was initiated at a basal infusion rate of $3 \mathrm{~mL} / \mathrm{h}$, patient-activated boluses of $3 \mathrm{~mL}$ with a 15-min lockout and a $15 \mathrm{~mL}$ 7-hour limit.

Femoral nerve catheters were placed perioperatively. The groin area was cleaned and draped in sterile fashion for the procedure. The skin at the needle entry site was infiltrated with $2 \mathrm{~mL}$ of lidocaine 1\% using a 25-gauge, $38 \mathrm{~mm}$ hypodermic needle. A $40 \mathrm{~mm}$ 18-gauge stimulating Tuohy needle connected to the negative lead of a constant voltage nerve stimulator (Stimuplex DIG ${ }^{\circledR}$; B-Braun/McGaw Medical, Bethlehem, PA) was inserted at the inguinal crease using ultrasound guidance. The stimulation frequency was set at 2 $\mathrm{Hz}$, a pulse width of $100 \mu$ s and a stimulating current of $1 \mathrm{~mA}$. When a QC was elicited, a 20-gauge stimulating catheter (StimuCath ${ }^{\circledR}$; Arrow International, Reading, PA, USA) connected to the nerve stimulator was inserted through the needle and advanced while maintaining an EMR until resistance was met. When a QC was not maintained after advancement, the catheter and needle were withdrawn, the needle repositioned and the catheter re-advanced. After two attempts of needle repositioning, the ensuing QC or sartorius muscle contraction (SC) motor response was accepted. The Tuohy needle was then withdrawn and the catheter was capped (Snaplock ${ }^{\mathrm{TM}}$, Arrow International, Reading, PA, USA). The nerve stimulator was reconnected and the threshold current to elicit a motor response was measured. The time from Tuohy needle insertion to catheter placement, the EMR, and the threshold current were recorded. The catheter was secured by subcutaneous tunneling $50 \mathrm{~mm}$ lateral to the insertion site. Patients that failed to obtain surgical anesthesia from the neuraxial block were given local anesthesia via the femoral catheter in the immediate postoperative period and excluded from data analysis. In the remaining subjects the catheter remained capped until local anesthetic administration the following morning.

Patients received a dose of warfarin (2.5 to $5 \mathrm{mg}$ ) following surgery on POD 0. Epidural analgesia was discontinued early on the morning of POD 1. Following the resolution of the epidural analgesia and after visual inspection of the femoral nerve catheter for dislodgement, the catheter was connected to a nerve stimulator set at a frequency of $2 \mathrm{~Hz}$, pulse width of $100 \mu \mathrm{s}$, and current of $1 \mathrm{~mA}$, and the EMR was recorded. The current was decreased or increased in $0.1 \mathrm{~mA}$ increments up to $5 \mathrm{~mA}$ until the minimum current threshold for motor response was detected. A test dose (3 $\mathrm{mL}$ ) of lidocaine $45 \mathrm{mg}$ with epinephrine $15 \mu \mathrm{g}$ was then administered through the femoral nerve catheter and patients were monitored for intravascular injection. If the minimum current threshold eliciting a motor response was $\leq 0.80 \mathrm{~mA}, 10 \mathrm{~mL}$ of $0.25 \%(25 \mathrm{mg})$ of ropivacaine was administered in $5 \mathrm{~mL}$ incremental doses through the catheter. If the minimum threshold current was higher $(>0.8 \mathrm{~mA})$ or if no motor response was elicited, $20 \mathrm{~mL}$ of $0.25 \%$ (50 mg) of ropivacaine was administered as above. Patients were assessed using a 21-gauge needle for pinprick sensory anesthesia of the femoral nerve distribution (middle third of the medial aspect of the thigh and the anteromedial middle third of the leg) by an observer unaware of the dose administered, at 5-minute intervals for 30 minutes. ${ }^{3}$ Femoral nerve blockade was considered 'complete' if there was a lack of pinprick sensation in the femoral nerve distribution of the thigh and calf within 30 minutes. The block was considered 'delayed' if sensory anesthesia was decreased but not absent or if there was no loss of sensation within 30 minutes. After 30 minutes of observation, the catheter was then attached to an infusion pump set to deliver $5 \mathrm{mg}$ of ropivacaine $0.1 \%$ per hour. 
On the morning of POD 2, the ropivacaine infusion was discontinued and the femoral catheter was removed. The catheter insertion site was evaluated for bruising and bleeding and the patients were questioned regarding the presence of numbness, tingling, or pain in the distribution of the femoral nerve both at the time of removal of the catheter and prior to discharge. Verbal rating scores for pain $(0-10)$ and opioid analgesics administered were recorded during POD 1. At discharge, patients were also asked to rate their satisfaction with anesthesia and postoperative analgesia on a 10 -point scale $(0=$ not satisfied to $10=$ highly satisfied $)$. They were also asked if they would recommend this method of pain management.

\section{Statistical analysis}

Interval data (age, catheter placement time, minimum current capture threshold, verbal rating pain scores, and opioid consumption) and ordinal data (subject satisfaction) were compared using the Mann-Whitney $U$-test. Binomial data (gender, EMR) were compared using a $\chi^{2}$ statistic. Sensitivity and specificity of an elicited EMR of PS at the time of catheter placement and prior to local anesthetic administration with a successful block at 30 minutes were determined by constructing $2 \times 2$ cross-tabulation tables. The overall predictive power of the threshold current at catheter placement and prior to local anesthetic administration with a successful block at 30 minutes was determined by constructing receiver operator characteristics (ROC) curves of the sensitivity against 1 - specificity and calculating the area under the curve (AUC). AUCs were compared for equivalence using nonparametric methods. The optimal current threshold was determined as the point of intersection of the lines of sensitivity and specificity. Comparison of the equivalence of sensitivity and specificity of the elicited EMRs and the current threshold at the optimal cut-off value were made using the Gart and Nam's score method with skewness correction. Statistical tests were performed using PASW statistics version 18.0.2, release date April 2, 2010 (SPSS, Inc., Chicago, IL) and NCSS 2007 version 7.1.20, release date February 19, 2010 (NCSS, Inc., Kaysville, UT).

\section{Results}

One hundred patients were consented and 90 patients completed the study protocol. Ten patients were excluded from the analysis. Nine subjects had local anesthetic administered just after catheter placement owing to inadequate neuraxial anesthesia, and in one case data was not recorded prior to local anesthetic administration. Subject characteristics are
Table I Subject characteristics

\begin{tabular}{ll}
\hline Age $(\mathrm{y})$ & $65(58-73)$ \\
Height $(\mathrm{cm})$ & $167(163-173)$ \\
Weight $(\mathrm{kg})$ & $84(68-98)$ \\
Gender (M/F) & $28 / 63$ \\
Side of catheter placement (Left/Right) & $43 / 47$ \\
Needle depth at insertion of catheter $(\mathrm{cm})$ & $3(3-4)$ \\
Catheter insertion length (cm) & $15(14-17)$ \\
Catheter placement time (sec) & $360(300-457)$ \\
Time from catheter placement to drug & $19(18-21)$ \\
administration (h) &
\end{tabular}

Notes: Data displayed as median (interquartile range), or number of subjects.

summarized in Table 1 . Thirty minutes after ropivacaine injection complete loss of pinprick sensation was achieved in 63 patients, 25 had reduced pinprick sensation, and 2 had no loss in sensation.

The first elicited EMR after needle insertion was a QC in 52 patients and an SC in 38 patients (Figure 1). Needle redirection resulted in a $\mathrm{QC}$ in 89 of the 90 patients prior to catheter insertion. After advancing the catheter, 77 patients maintained a QC and 13 demonstrated an SC. A successful block was achieved in 57/77 subjects that had a QC and in 6/13 that had an SC at catheter placement $(P=0.054)$. A QC contraction at catheter placement had a sensitivity of 0.90 (95\% confidence interval [CI]: 0.81 to 0.96$)$ and a specificity of 0.26 (95\% [CI]: 0.13 to 0.45 ) for predicting a complete femoral nerve block.

The median interquartile range [IQR] threshold current was 0.50 ( 0.33 to 68$) \mathrm{mA}$ after catheter placement. In patients demonstrating a QC after catheter placement, the median (IQR) current threshold was 0.50 (0.30 to 0.68$) \mathrm{mA}$ and was not different from patients that demonstrated an SC 0.52 ( 0.44 to 1.1) $\mathrm{mA}(P=0.28)$. ROC analysis for block success identified an optimal cut-off current threshold of $0.50 \mathrm{~mA}$ and an area under the ROC curve of 0.45 ( $95 \%$ CI: 0.32 to 0.59 ) at catheter placement (Figure 2). Sensitivity and specificity were 0.51 (95\% CI: 0.39 to 0.63 ) and 0.41 (95\% CI: 0.55 to 0.83 ), respectively. There was no difference in the median (IQR) current threshold at the time of catheter placement in patients that had complete block 0.50 ( 0.40 to 0.67 ) $\mathrm{mA}$ compared with those with a delayed block $0.48(0.26$ to 0.68$) \mathrm{mA}(P=0.48)$.

Prior to drug administration a QC was obtained in 66 patients, 13 demonstrated an SC, and no motor response was obtained in 11 patients. Five of the 66 patients that demonstrated a QC had an SC at catheter placement, and 6 of the 13 patients that demonstrated an SC had a QC after catheter placement $(P<0.01)$. A QC contraction prior to drug administration had a sensitivity of 0.85 (95\% CI: 0.75 to 0.92$)$ and a specificity of 0.55 (95\% CI: 0.37 to 0.72 ) for predicting a complete femoral 


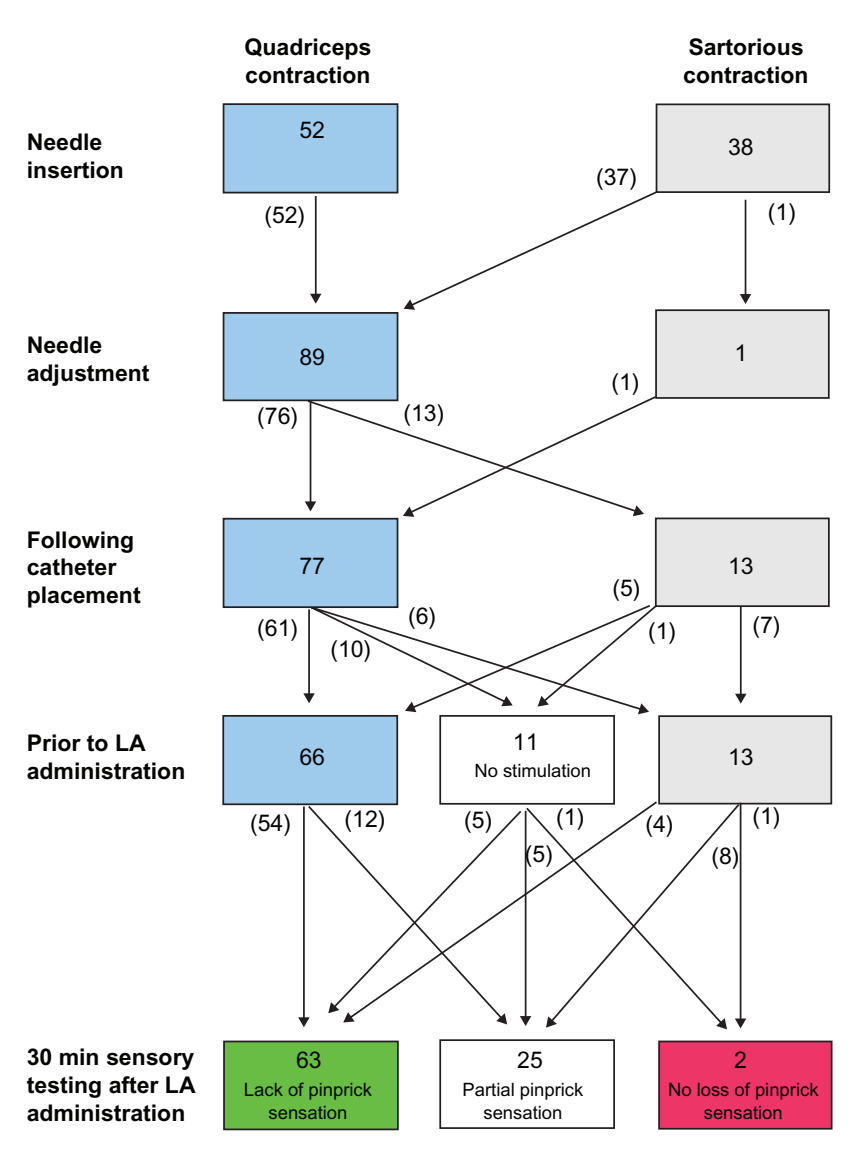

Figure I Pattern of elicited motor responses at study time points. Abbreviation: LA, local anesthetic.

nerve block. The specificity of a $\mathrm{QC}$ prior to drug administration was greater than at catheter placement $(P=0.006)$.

Compared to the threshold current at catheter placement, 27 patients had a decrease, 60 had an increase, and 3 exhibited no change in the threshold current prior to drug administration $(P<0.01)$. The median (IQR) threshold current in patients that achieved a complete block was $0.56(0.29$ to 0.80$) \mathrm{mA}$ compared with $1.1 \mathrm{~mA}(0.41$ to 2.75$) \mathrm{mA}$ for incomplete blocks $(P<0.01)$. The overall predictive value (area under the ROC curve) for the minimal current threshold prior to local anesthetic administration (0.74) was greater than that for the threshold current at catheter placement $(0.45)(P<0.001)$. The intersection of sensitivity and specificity for the minimal threshold current prior to local anesthetic injection was $0.84 \mathrm{~mA}$ (Figure 2). The sensitivity and specificity of current thresholds $\leq 0.84 \mathrm{~mA}$ was 0.87 ( $95 \%$ confidence interval 0.76 to 0.94$)$ and 0.55 (95\% confidence interval 0.39 to 0.71$)$ which were not different from the EMR responses at the same time. A successful block was achieved in all cases where a current threshold $\leq 0.25 \mathrm{~mA}$ was obtained. Within the current threshold groups, there was no difference in the proportion of patients that had complete block with an EMR of QC compared with SC; however in patients with a QC, 44 of 50 at a threshold
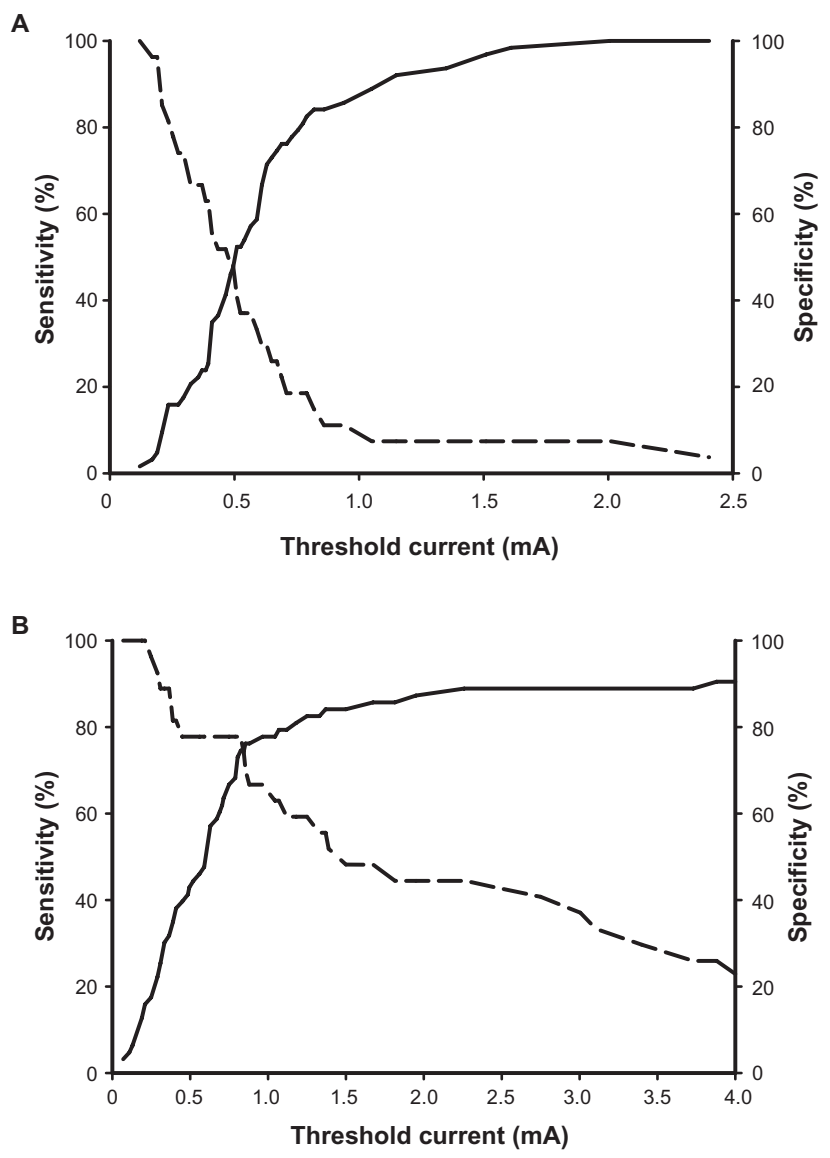

Figure 2 Sensitivity (solid line) and specificity (dashed line) of current threshold for predicting complete femoral analgesia.

Abbreviations: A, at catheter placement; B, prior to drug administration.

current $\leq 0.84 \mathrm{~mA}$ achieved a successful block compared to 10 of 16 with a threshold current $>0.84 \mathrm{~mA}(P=0.03)$.

Patients that achieved a complete block at 25 minutes reported a higher median (IQR) satisfaction with the analgesia management score of 9 (8 to 10) compared to patients with delayed analgesia score of 8 ( 7 to 9$)(P<0.01)$. Ninety-five percent of patients stated that they would recommend this method of analgesia to others. Verbal rating pain scores were not statistically different. No subject demonstrated clinically significant bruising or bleeding at the catheter site nor reported the presence of numbness, tingling, or pain in the distribution of the femoral nerve at discharge.

\section{Discussion}

When used to guide catheter positioning at placement, maintenance of a QC response with stimulating catheters has been reported to increase block success and reduce local anesthetic requirements compared to nonstimulating catheters. ${ }^{4,5}$ Our findings extend the evidence supporting the use of stimulating catheters for continuous femoral nerve blocks when a time interval from the placement of the catheter to the drug administration will occur. At the time of drug administration the re-evaluation of the current threshold and elicited motor 
Table 2 Complete block by elicited motor response at optimal threshold current cut-off value

\begin{tabular}{|c|c|c|c|c|}
\hline \multicolumn{2}{|l|}{ Threshold current $\leq 0.84 \mathrm{~mA}(n=54)$} & \multicolumn{3}{|c|}{$\boldsymbol{P}$} \\
\hline Motor response & Complete block & Delayed block & & \\
\hline Quadriceps contraction & 45 & 6 & & \\
\hline Sartorius muscle & 2 & I & & \\
\hline Total & 47 & 7 & & \\
\hline Threshold current $>0.84 \mathrm{~mA}(n=36)$ & & & & \\
\hline Quadriceps contraction & 9 & 7 & & $<0.0005^{\dagger}$ \\
\hline Sartorius muscle/No response & 6 & 14 & & \\
\hline Total & 15 & 21 & & \\
\hline
\end{tabular}

Notes: Current threshold cut-off value $(0.84 \mathrm{~mA})$ determined by receiver operator characteristics analysis at the intersection of sensitivity and specificity. Data displayed as number of subjects. $*=$ comparison of block success between elicited motor response within threshold current group. P-value ${ }^{\dagger}=$ comparison of block success rate between threshold current groups.

response demonstrated increased sensitivity and greater specificity for complete block compared with those recorded at placement.

Despite eliciting a QC in $99 \%$ of the patients just prior to catheter placement, an EMR of QC was only maintained with advancing the catheter in $85 \%$ of the patients despite two attempts at repositioning. Median current thresholds were similar with either an EMR of QC or SC after catheter placement, suggesting that a similar distance from the nerve to the catheter tip was achieved. Nonetheless, there was a significant change in the EMR at catheter placement compared with those observed prior to drug administration. Current threshold increased between catheter placement and drug administration in the majority of cases. Taken together our data suggests that there is considerable variability in the catheter-to-nerve relationship and possibly there are changes in the distance from the catheter to the nerve that occur in the perioperative period. Conductivity changes in the tissues surrounding the nerve due to edema may have also contributed to our findings.

Our study demonstrated that a current threshold of $\leq 0.84$ and an EMR of QC elicited at catheter placement and prior to drug administration are highly sensitive for predicting a complete block.

At the time of this study it was our practice to use epidural analgesia for the first 24 hours following surgery to provide analgesia to the anterior and posterior aspects of the knee. Patients received warfarin the night of surgery as part of a standard thromboembolism prophylaxis protocol. The femoral nerve catheters were placed in the perioperative period before warfarin was administered but the infusion of local anesthetic was delayed until after the epidural catheter was removed to prevent profound quadriceps weakness and possible delay in initiating physical therapy. In this way an additional procedure was not required to initiate femoral analgesia on the day after surgery. Although this practice may not be common, it allowed for reassessment of the EMR and current threshold at a time point after placement to assess the utility of restimulation in situ.

Previous investigators have suggested using lower stimulating currents when positioning femoral nerve catheters and threading the catheters only 4-5 cm beyond the needle. ${ }^{7,8}$ We used a stimulating current of $1 \mathrm{~mA}$ to identify the nerve when placing the femoral catheter and threaded the catheters $8-20 \mathrm{~cm}$ from the inguinal crease. Increasing the length of the catheter that is threaded may have also increased the variability in the catheter-to-the-nerve proximity, but complete blockade of the lumbar plexus is more likely when the catheter is advanced $15-20 \mathrm{~cm}$ proximally with the tip within the psoas sheath. ${ }^{6}$ We chose to advance the catheters until resistance was met because in our experience this practice prevents catheter dislodgement throughout surgery or in the postoperative period during physical therapy as well as minimizing the incidence of leakage.

In a previous study we demonstrated the relationship between ultrasound imaging and eliciting motor response during femoral nerve stimulation. ${ }^{7}$ Although visualization of the needle tip may initially allow us to determine the distance between the femoral nerve and the Touhy needle, it may be difficult to estimate the distance from the stimulating catheter tip to the femoral nerve while advancing the catheter. In addition, the pattern of local anesthetic distribution visualized by ultrasonography around the 
femoral catheter tip and femoral nerve, and its correlation with the stimulating current is an important parameter that should be investigated in the future studies.

There are several limitations to our study: The volume of the local anesthetic solution was not the same in the two groups, although the volumes $(10-20 \mathrm{~mL})$ of ropivacaine administered were similar to dosages used in other studies. ${ }^{8}$ We arbitrarily set a current threshold of $0.8 \mathrm{~mA}$ for determining the dose of ropivacaine, which may have affected the success rate compared with a single dose. We chose this value as the cut-off point for determining local anesthetic dose in concordance with the upper limit of the acceptable threshold used in other studies while advancing stimulating catheters. ${ }^{9,10}$ Whether the current threshold of $<0.80 \mathrm{~mA}$ reflects a catheter tip position below the fascia iliaca thus minimizing the diffusion barrier between the local anesthetics and the nerve remains to be studied. An EMR could not be elicited despite a current of $5 \mathrm{~mA}$ in 11 patients on POD 1; however, a complete block was achieved following $50 \mathrm{mg}$ of ropivacaine in five of these patients. This may suggest that in these patients the lack of an EMR may not be a limitation to achieving complete anesthesia via these catheters. Although all subjects had normal sensory assessment prior to EMR and threshold evaluations postoperatively, we cannot rule out that residual epidural analgesia may have confounded the results of our study. Finally, we did not investigate the relationship between time of placement and change in EMR or current threshold. The median time interval in this study was 19 hours and shorter intervals may have influenced the difference in EMR and current threshold seen in this study.

In conclusion, the elicited motor response and threshold current from an in situ femoral catheter measured prior to local anesthetic injection when there is a time delay from insertion to drug administration are important determinants of the success of femoral nerve block. This finding has important clinical implications as satisfaction with pain management was greater when rapid anesthesia was obtained after bolus administration. Further studies to assess the value of catheter manipulation to achieve a desired EMR of reduced current threshold of the in situ catheter are warranted.

\section{Disclosure}

The authors have no conflicts of interest to disclose. The authors received departmental financial support.

Presented in part at the 2007 Annual Meeting of the American Society of Anesthesiologists, San Francisco, CA.

\section{References}

1. Singelyn FJ, Deyaert M, Joris D, Pendeville E, Gouverneur JM. Effects of intravenous patient-controlled analgesia with morphine, continuous epidural analgesia, and continuous three-in-one block on postoperative pain and knee rehabilitation after unilateral total knee arthroplasty. Anesth Analg. 1998;87:88-92.

2. Ilfeld BM, Thannikary LJ, Morey TE, Vander Griend RA, Enneking FK. Popliteal sciatic perineural local anesthetic infusion. Anesthesiology. 2004;101:970-977.

3. Jochum D, O'Neill T, Jabbour H, Diarra PD, Cuignet-Pourel E, Bouaziz H. Evaluation of femoral nerve blockade following inguinal paravascular block of Winnie: are there still lessons to be learnt? Anaesthesia. 2005;60:974-977.

4. Wehling MJ, Koorn R, Leddell C, Boezaart A. Electrical nerve stimulation using a stimulating catheter: what is the lower limit? Reg Anesth Pain Med. 2004;29:230-233.

5. Cuvillon P, Ripart J, Lalourcey L, et al. The continuous femoral nerve block catheter for postoperative analgesia: bacterial colonization, infectious rate and adverse effects. Anesth Analg. 2001;93:1045-1049.

6. Capdevila X, Biboulet P, Morau D, et al. Continuous three-in one block for postoperative pain after lower limb orthopedic surgery: where do the catheters go? Anesth Analg. 2002;94:1001-1006.

7. Nader A, Malik K, Kendall MC, Benzon H, McCarthy RJ. Relationship between ultrasound imaging and eliciting motor response during femoral nerve stimulation. J Ultrasound Med. 2009;28:345-350.

8. Weber A, Fournier R, Riand N, Gamulin Z. Duration of analgesia is similar when $15,20,25$, and $30 \mathrm{ml}$ of ropivacaine $0.5 \%$ are administered via a femoral catheter. Can J Anesth. 2005;52:390-396.

9. Salinas F, Neal JM, Sueda LA, Kopacz DJ, Liu SS. Prospective comparison of continuous femoral nerve block with non-stimulating catheter placement versus stimulating catheter-guided perineural placement in volunteers. Reg Anesth Pain Med. 2004;29:212-220.

10. Morin AM, Eberhart LH, Behnke HK, et al. Does the femoral nerve catheter placement with stimulating catheters improve effective placement? A randomized, controlled, and observer-blinded trial. Anesth Analg. 2005;100:1503-1510.
Local and Regional Anesthesia

\section{Publish your work in this journal}

Local and Regional Anesthesia is an international, peer-reviewed, open access journal publishing on the development, pharmacology, delivery and targeting and clinical use of local and regional anesthetics and analgesics. The journal welcomes submitted papers covering original research, basic science, clinical studies, reviews \& evaluations,

\section{Dovepress}

guidelines, expert opinion and commentary, case reports and extended reports. The manuscript management system is completely online and includes a very quick and fair peer-review system, which is all easy to use. Visit http://www.dovepress.com/testimonials.php to read real quotes from published authors. 